\title{
Dynamic Contrast-enhanced Magnetic Resonance Imaging for Patients with Breast Cancer Receiving First-line Bevacizumab and Paclitaxel
}

\author{
KATSUHIKO NAKATSUKASA ${ }^{1}$, YOSHIMI OUCHI ${ }^{1}$, KOUICHI SAKAGUCHI $^{1}$, \\ MARI GOTO $^{2}$, EIICHI KONISHI ${ }^{3}$ and TETSUYA TAGUCHI ${ }^{1}$ \\ Departments of ${ }^{1}$ Endocrine and Breast Surgery, \\ ${ }^{2}$ Radiology and ${ }^{3}$ Surgical Pathology Kyoto Prefectural University of Medicine, Kyoto, Japan
}

\begin{abstract}
Background: Bevacizumab plus paclitaxel shows promise for metastatic disease; however, there is no predictive biomarker. Dynamic contrast-enhanced (DCE) magnetic resonance imaging (MRI) is widely used to evaluate the efficacy of anti-angiogenic therapies. Patients and Methods: Patients with locally advanced/metastatic breast cancer who had not received any prior chemotherapy or hormone therapy were included. DCE-MRI was conducted at baseline and after one and two cycles of bevacizumab and paclitaxel. The percentage change in the volume transfer constant (AKtrans) and the correlation of $\Delta$ Ktrans with tumour regression and time to progression (TTP) were evaluated. Results: The mean AKtrans from baseline after one and two cycles was $-51.4 \%$ and $-55.1 \%$, respectively. Patients with $\Delta$ Ktrans $\geq 50 \%$ displayed more tumour regression than those with $\triangle$ Ktrans $<50 \%$; TTP was not significantly different. Conclusion: We demonstrate a decrease in blood permeability following bevacizumab and paclitaxel using DCE-MRI and a correlation between $\triangle$ Ktrans and tumour regression.
\end{abstract}

Bevacizumab is a humanized monoclonal antibody to vascular endothelial growth factor (VEGF) that recognizes all isoforms of VEGFA. Preclinical models have shown inhibition of angiogenesis and regression of solid tumors in response to VEGF monoclonal antibodies alone or to combination chemotherapy (1-3).

Correspondence to: Katsuhiko Nakatsukasa, MD, Department of Endocrine and Breast Surgery, Kyoto Prefectural University of Medicine, 465 Kajii-cho, Kawaramachi-hirokoji, Kamigyo-ku, Kyoto City 602-8566, Japan. Tel: +81 752515534, Fax: +81 752510270, e-mail: kacchan@koto.kpu-m.ac.jp

Key Words: Advanced breast cancer, DCE-MRI, bevacizumab, paclitaxel.
Paclitaxel binds to $\beta$-tubulin and stabilizes microtubules, repressing the dynamic instability of spindle microtubules and thereby blocking the cell cycle at the metaphase-toanaphase transition (4). Compared to paclitaxel alone, bevacizumab combined with paclitaxel as first-line treatment significantly prolongs progression-free survival in metastatic breast cancer (5-10). Bevacizumab combined with paclitaxel is a promising therapy in the metastatic setting, but it does not yet have a predictive biomarker for response (11).

Dynamic contrast-enhanced (DCE) magnetic resonance imaging (MRI) has been extensively used in pre-clinical and clinical trials to evaluate the efficacy of antiangiogenic therapy. Parameters of functional tumour microvascular, such as vascular volume and vascular permeability, are derived from the pharmacokinetic modelling of T1-weighted DCE-MRI time-course data and used as surrogate biomarkers of treatment efficacy (12, 13). DCE-MRI is a non-invasive and rapid (requires approximately $5 \mathrm{~min}$ ) imaging technique that can assess tumour perfusion, microvascular vessel wall permeability, and extravascular/extracellular volume fraction $(14,15)$. Pharmacokinetic parameters are assessed using 3-Tesla DCE-MRI. If changes in DCE-MRI findings measured during the treatment were found to correlate with disease outcomes, they could be developed as predictive biomarkers for angiogenic activity in tumours. One DCEMRI parameter, the volume transfer constant ( $\Delta$ Ktrans), has been reported to be a pharmacodynamic and predictive biomarker for bevacizumab regimens in metastatic colorectal cancer (16). However, few studies have shown any correlation between parameter changes and clinical outcomes during paclitaxel and bevacizumab therapy of metastatic breast cancer. Herein, we report for the first time a correlation between changes in blood permeability and a decrease in tumour volume using 3-Tesla DCE-MRI in Japanese patients with metastatic breast cancer. 


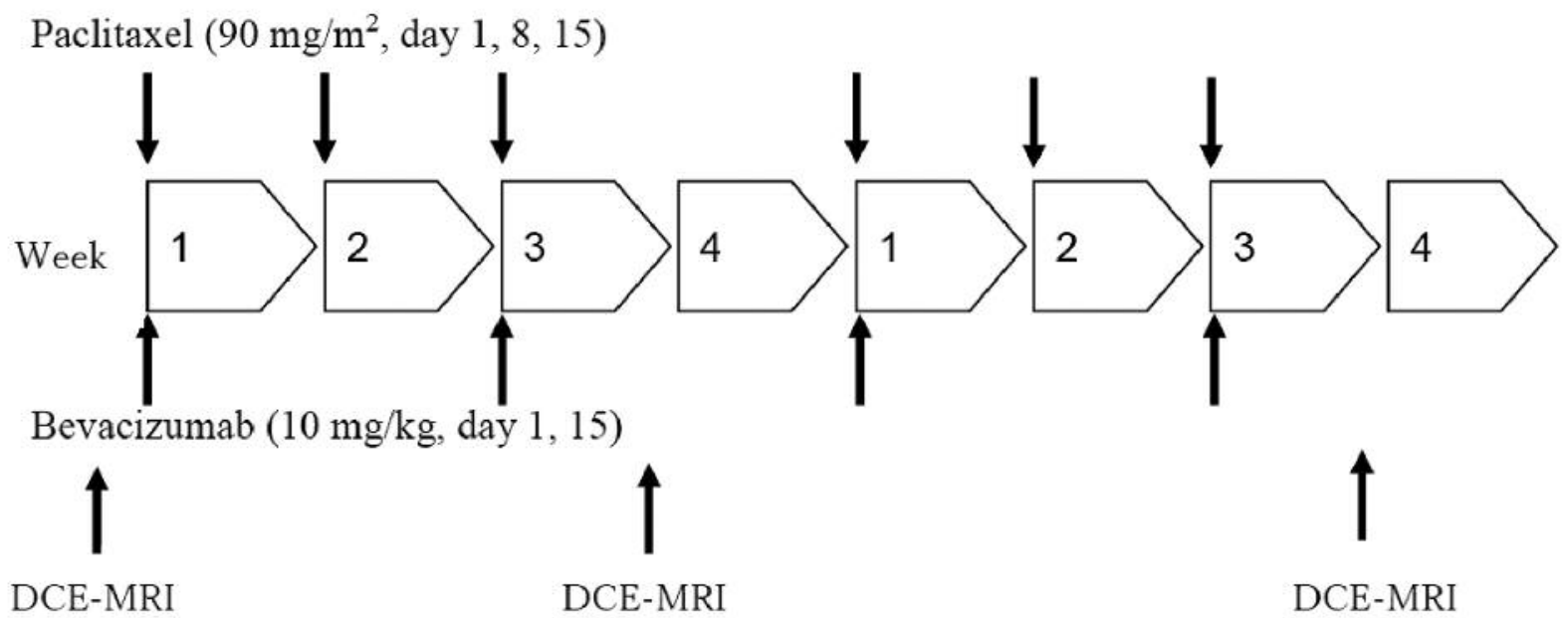

Figure 1. Study scheme. DCE-MRI, Dynamic contrast-enhanced magnetic resonance imaging.

\section{Patients and Methods}

Patients. Patients were selected based on the following eligibility criteria: (i) Age $>20$ and $<80$ years; (ii) performance status (Eastern Cooperative Oncology Group scale) score 0-1 (iii); expected survival period of $>6$ months; (iv) no residual effects from prior chemotherapy; (v) adequate organ function (white blood cell count $\geq 4,000 / \mathrm{mm}^{3}$, neutrophil count $\geq 2,000 / \mathrm{mm}^{3}$, haemoglobin concentration $\geq 9.0 \mathrm{~g} / \mathrm{dI}$, platelet count $\geq 100,000 / \mathrm{mm}^{3}$, total bilirubin $\leq 1.5 \mathrm{mg} / \mathrm{dI}$, aspartate transaminase concentration $\leq 100 \mathrm{IU} / \mathrm{I}$, alanine transaminase concentration $\leq 100 \mathrm{IU} / \mathrm{I}$, and creatine concentration $\geq 60 \mathrm{mI} / \mathrm{min}$ ); and (vi) written informed consent provided. The main exclusion criteria were as follows: (i) Pregnant or possibility of pregnancy; (ii) active breast cancer; (iii) severe complications; (iv) uncontrolled pleural effusion, ascites, or oedema; (v) brain metastasis; or (vi) physician's decision not to register the patient in this study.

Study design. This trial was a non-randomized prospective study evaluating bevacizumab combined with paclitaxel as the first-line therapy for human oestrogen receptor-2 (HER2)-negative locally advanced/metastatic breast cancer (LA/mBC). Patients with HER2negative $\mathrm{LA} / \mathrm{mBC}$ who had received no prior chemotherapy for $\mathrm{LA} / \mathrm{mBC}$ received $10 \mathrm{mg} / \mathrm{kg}$ bevacizumab on days 1 and 15 and 10 $\mathrm{mg} / \mathrm{kg}$ bevacizumab combined with $90 \mathrm{mg} / \mathrm{m}^{2}$ paclitaxel on days 1 , 8 , and 15 , repeated every 4 weeks until disease progression, unacceptable toxicity, or patient/physician decision. The study scheme is shown in Figure 1. All patients provided their written informed consent before enrolling in the study. The study was approved by the Institutional Review Board of Kyoto Prefectural University of Medicine on September 13, 2013 (ERB-C-78) and was registered with the UMIN Clinical Trial Registry (UMIN-CTR, UMIN000021677). Because patient recruitment was difficult and this study was completed on March 31, 2018, only nine patients were registered.

DCE-MRI study protocol. Breast MRI was performed with 3-T MRI scanners (MAGNETOM Skyra; Siemens Healthcare, Erlangen,
Germany) using a 16-channel bilateral breast coil. Patients were positioned in the prone position with their arms up. The coil was adjusted to the breast size, but no compression was applied.

Firstly, two variable FA $(5,15$ degree) with Dixon were applied for T1 mapping; secondly, DCE-MRI was obtained in two parts: early phase ( 25 frames within $2 \mathrm{~min} 22 \mathrm{~s}$, started $22 \mathrm{~s}$ before contrast material injection) and delayed phase (nine frames within $30 \mathrm{~s}$, started $5 \mathrm{~min}$ after contrast injection) for estimation of pharmacokinetic parameters. DCE-MRI was performed using timeresolved angiography with interleaved stochastic trajectories (TWIST)-volume-interpolated breath-hold examination (VIBE). The following imaging parameters were applied: repetition time ms/echo time ms, 5.6/2.5; field of view, $360 \mathrm{~mm}$; matrix, $269 \times 384$ and 60 slices; parallel imaging factor, 3 ; and fat suppression, two-point Dixon. This resulted in a temporal resolution of $4.7 \mathrm{~s}$ for each phase except for the first TWIST frame (22 s, full k-space sampling).

Contrast agent $[0.1 \mathrm{mmol} / \mathrm{kg}$ Dotarem $(0.5 \mathrm{mmol} / \mathrm{ml}$ gadoteric acid); Guerbet Japan, Tokyo, Japan] was administered through an intravenous cannula placed in the cubital vein. The power injector (Sonic Shot; Nemoto Kyorindo, Tokyo, Japan) was started at the beginning of the second phase of the TWIST-VIBE sequence at a dose of $0.1 \mathrm{mmol} / \mathrm{kg}$ and a rate of $2 \mathrm{ml} / \mathrm{s}$, followed by a $20-\mathrm{ml}$ saline flush.

DCE-MRI was conducted at baseline and after one and two cycles of chemotherapy and Ktrans values were measured.

DCE-MRI kinetic parameters. Pharmacokinetic maps were obtained using the commercial software Tissue 4D (Siemens Healthcare). Initially, motion correction was performed automatically. The arterial input function was automatically calculated based on population-based arterial input functions built in Tissue 4D. Finally, 3D maps of Ktrans were obtained. Then one rater (K.N., a breast surgeon with 15 years of experience) placed regions of interest on the Ktrans map for each lesion, avoiding visible necrosis, vessels, calcifications, and cystic-appearing areas, to generate the Ktrans value. The pharmacokinetic parameters were analyzed based using the Tofts model. 
Table I. Baseline characteristics patients with locally advanced/metastatic breast cancer receiving bevacizumab and paclitaxel.

\begin{tabular}{ll}
\hline Characteristic & VaIue (N=9) \\
\hline Median age (range), years & $61(43-79)$ \\
Clinical stage, $\mathrm{n}$ & \\
$\quad$ III b & 8 \\
IV & 1 \\
Histology, n & 9 \\
$\quad$ Invasive ductal carcinoma & 0 \\
$\quad$ Invasive lobular carcinoma & \\
Subtype, n & 3 \\
$\quad$ Luminal-A-like & 5 \\
$\quad$ Luminal-B-like & 1 \\
$\quad$ Triple-negative & \\
\hline
\end{tabular}

Luminal-A-Iike: Oestrogen receptor-positive and Ki-67 index $<20 \%$; luminal-B-like: oestrogen receptor-positive and Ki-67 index $\geq 20 \%$.

Assessment of outcomes. The primary endpoint was the percentage change in Ktrans ( $\Delta$ Ktrans) from baseline after one and two cycles of therapy. The secondary endpoints included the correlation of $\Delta$ Ktrans with tumour regression rate and time to progression (TTP), defined as the length of time from the date of the start of treatment until disease progression or spread to other parts of the body, and assessment of safety. We defined tumor regression rate as (maximum diameter before treatment - after one cycle) / maximum diameter before treatment* 100 .

Safety. Toxicity was assessed according to the second version of the National Cancer Institute Common Toxicity Criteria (NCI-CTC, v2).

Statistical analysis. Mann-Whitney $U$-test was performed to compare patients divided into two groups according to $\Delta$ Ktrans, using a cut-off of $50 \%$ ( $\Delta$ Ktrans $\geq 50 \%$ vs. $<50 \%)$. p-Values reported are based on a two-sided comparison, and values of 0.05 or less were considered statistically significant. TTP was examined using the Kaplan-Meier method. All statistical analyses were performed using the JMP software version 12 (SAS Institute Inc., Cary, NC, USA).

\section{Results}

Patient characteristics are summarized in Table I. A total of nine patients were enrolled in this study.

Ktrans values at baseline and after one and two cycles of chemotherapy are shown in Figure 2. The mean $\Delta$ Ktrans from baseline after one and two cycles of therapy was $-51.4 \%$ and $-55.1 \%$, respectively. The tumour regression rate according to $\Delta$ Ktrans was assessed using the Mann-Whitney $U$-test. The tumour regression rate in the five patients with $\Delta$ Ktrans $\geq 50 \%$ was significantly higher than that in the four patients with $\Delta$ Ktrans $<50 \%$ (43.1\% vs. $14.1 \%$; $p=0.028$ ).

TTP according to $\Delta$ Ktrans is shown in Figure 3 . TTP in the five patients with $\Delta \mathrm{K}$ trans $\geq 50 \%$ tended to be longer than that in the four patients with $\Delta$ Ktrans $<50 \%$ (median=359 vs. 158 days), although the difference was not

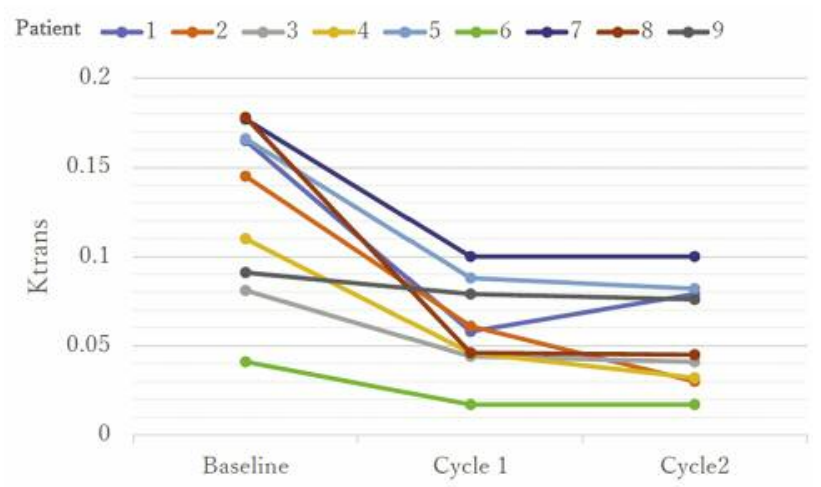

Figure 2. Volume transfer constant (Ktrans) at baseline and after one and two cycles of bevacizumab and paclitaxel in individual patients with locally advanced/metastatic breast cancer.

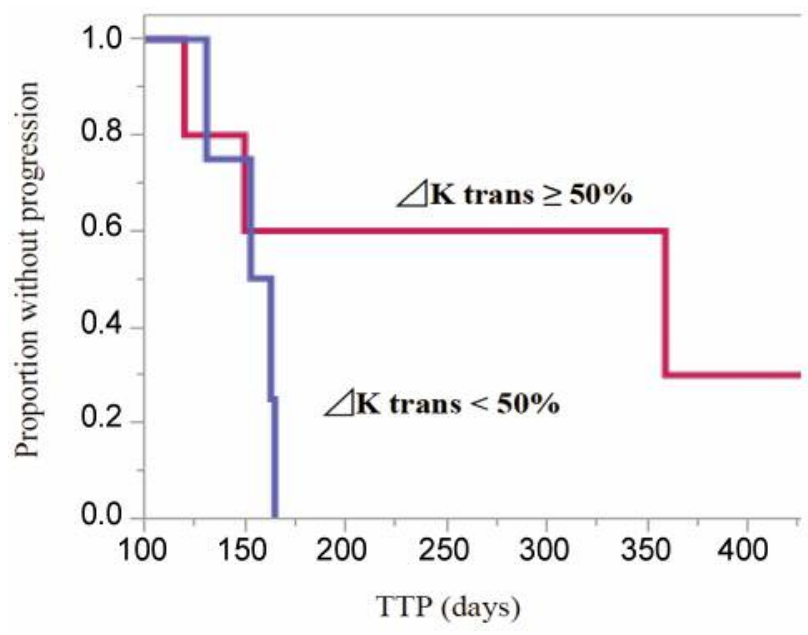

Figure 3. Time to progression (TTP) according to change in volume transfer constant (AKtrans) in patients with locally advanced/metastatic breast cancer receiving bevacizumab and paclitaxel. Median TTP was 359 days in those with $\triangle$ Ktrans $\geq 50 \%$ vs. 158 days in those with $<50 \%$ days) $(p=0.211)$.

significant $(p=0.21)$; the lack of significance was probably due to the small sample size.

Safety was evaluated in the nine patients treated with paclitaxel and bevacizumab. Adverse effects with an occurrence rate $\geq 5 \%$ are listed in Table II. Hypertension and proteinuria occurred in approximately $30 \%$ study participants, but almost all were grade 1 and controllable.

\section{Discussion}

In three randomized phase III trials, adding bevacizumab to first-line chemotherapy for HER2-negative $\mathrm{mBC}$ significantly improved the progression-free survival and 
Table II. Adverse events experienced by patients with locally advanced/metastatic breast cancer receiving bevacizumab and paclitaxel $(N=9)$. No patient experienced grade 3 or higher adverse events.

\begin{tabular}{lc}
\hline & Toxicity grade \\
$1 / 2$ \\
Adverse event & $\mathrm{n}(\%)$ \\
\hline Neutropenia & $2(22.2)$ \\
Fatigue & $1(11.1)$ \\
Nausea & $1(11.1)$ \\
Hypertension & $3(30.0)$ \\
Constipation & $1(11.1)$ \\
Peripheral neuropathy & $2(22.2)$ \\
Proteinuria & $3(30.0)$ \\
\hline
\end{tabular}

overall response rate, but not the overall survival (5-7). Numerous post-hoc retrospective subgroup analyses based on clinical and disease characteristics suggest that no specific subgroup derives a significantly greater benefit from bevacizumab $(17,18)$. Plasma VEGFA was considered to be the most promising candidate as a biomarker $(19,20)$, but results of the MERiDiAN trial failed to confirm this (11).

There is convincing evidence that DCE-MRI is a valuable tool for assessing response to neoadjuvant chemotherapy. One study that evaluated 33 patients with LABC with serial DCE-MRI showed that all the complete responders had an early tumour reduction of $>45 \%$ (21). In another study that assessed 62 patients with DCE-MRI before and after one cycle of neoadjuvant chemotherapy, the response map reliably predicted a complete pathological response, as well as disease-free and overall survival (22). Our findings, that show a correlation of $\Delta$ Ktrans with tumour reduction rate and TTP, are consistent with these results.

There are certain critical questions to be considered regarding the association between $\Delta K$ trans and prognosis. The first is whether $\Delta K$ trans in primary breast cancer is a better predictor than $\Delta$ Ktrans in metastatic sites such as the liver or lung. The second is whether the second MRI after the baseline DCE-MRI should be after one, two, or three cycles. The third is whether bevacizumab and paclitaxel should be replaced with other chemotherapeutic agents if $\Delta K$ trans does not decrease. To answer these questions, we are planning a head-to-head randomized clinical trial in which patients in whom $\Delta$ Ktrans decreases by $\geq 50 \%$ will continue receiving bevacizumab and paclitaxel, whereas those in whom it decreases by $<50 \%$ will be administered other therapies.

Although the findings of this study are significant, there exist several limitations. Firstly, this was a non-randomized study, and thus we were limited by the data available and unable to collect additional data. However, the $\Delta$ Ktrans data are reliable since all the patients were receiving bevacizumab and paclitaxel as first-line therapy and all DCE-MRI testing was conducted at a single institution. The second limitation is the small sample size of the study. A larger sample size could have provided more reliable results.

In summary, as far as we are aware of, we are the first to use DCE-MRI to show that blood permeability in $\mathrm{LA} / \mathrm{mBC}$ decreases following first-line bevacizumab and paclitaxel treatment. We also showed a correlation between $\Delta$ Ktrans and tumour regression rate.

\section{Conflicts of Interest}

The Authors have no conflicts of interest to declare in regard to this study.

\section{Funding}

There was no funding for this study.

\section{Acknowledgements}

The Authors thank all the patients and investigators who participated in this study.

This study was presented at the 24th Annual Meeting of the Japanese Breast Cancer Society, Fukuoka, July 13-15, 2017 (Presentation No. PS-2-20-04).

\section{References}

1 Kim KJ, Li B, Winer J, Armanini M, Gillett N, Phillips HS, and Ferrara N: Inhibition of vascular endothelial growth factorinduced angiogenesis suppresses tumour growth in vivo. Nature 362: 841-844, 1993.

2 Borgstrom P, Gold DP, Hillan KJ and Ferrara N: Importance of VEGF for breast cancer angiogenesis in vivo: Implications from intravital microscopy of combination treatments with an antiVEGF neutralizing monoclonal antibody and doxorubicin. Anticancer Res 19: 4203-4214, 1999.

3 Millauer B, Shawver LK, Plate KH, Risau W and Ullrich A: Glioblastoma growth inhibited in vivo by a dominant-negative Flk-1 mutant. Nature 367: 576-579, 1994.

4 Horwitz SB: Mechanism of action of taxol. Trends Pharmacol Sci 13: 134-136, 1992.

5 Miller K, Wang M, Gralow J, Dickler M, Cobleigh M, Perez EA, Shenkier T, Cella D and Davidson NE: Paclitaxel plus bevacizumab versus paclitaxel alone for metastatic breast cancer. N Engl J Med 357: 2666-2676, 2007.

6 Miles DW, Chan A, Dirix LY, Cortés J, Pivot X, Tomczak P, Delozier T, Sohn JH, Provencher L, Puglisi F, Harbeck N, Steger GG, Schneeweiss A, Wardley AM, Chlistalla A and Romieu G: Phase III study of bevacizumab plus docetaxel compared with placebo plus docetaxel for the first-line treatment of human epidermal growth factor receptor 2-negative metastatic breast cancer. J Clin Oncol 28: 3239-3247, 2010.

7 Robert NJ, Diéras V, Glaspy J, Brufsky AM, Bondarenko I, Lipatov ON, Perez EA, Yardley DA, Chan SY, Zhou X, Phan SC and O'Shaughnessy J: RIBBON-1: randomized, double-blind, 
placebo-controlled, phase III trial of chemotherapy with or without bevacizumab for first-line treatment of human epidermal growth factor receptor 2-negative, locally recurrent or metastatic breast cancer. J Clin Oncol 29: 1252-1260, 2011.

8 Rossari JR, Metzger-Filho O and Paesmans M, Saini KS, Gennari A, de Azambuja E and Piccart-Gebhart: M Bevacizumab and breast cancer: A meta-analysis of first-line phase III studies and a critical reappraisal of available evidence. J Oncol 2012: 417673, 2012.

9 Aogi K, Masuda N, Ohno S, Oda T, Iwata H, Kashiwaba M, Fujiwara Y, Kamigaki S, Ito Y, Ueno T and Takashima S: Firstline bevacizumab in combination with weekly paclitaxel for metastatic breast cancer: efficacy and safety results from a large, open-label, single-arm Japanese study. Breast Cancer Res Treat 129: 829-838, 2011.

10 Brufsky AM, Hurvitz S, Perez E, Swamy R, Valero V, O’Neill $\mathrm{V}$ and Rugo HS: RIBBON-2: a randomized, double-blind, placebo-controlled, phase III trial evaluating the efficacy and safety of bevacizumab in combination with chemotherapy for second-line treatment of human epidermal growth factor receptor 2-negative metastatic breast cancer. J Clin Oncol 29: 4286-4293, 2011.

11 Miles D, Cameron D, Bondarenko I, Manzyuk L, Alcedo JC, Lopez RI, Im SA, Canon JL, Shparyk Y, Yardley DA, Masuda N, Ro J, Denduluri N, Hubeaux S, Quah C, Bais C and O'Shaughnessy J: Bevacizumab plus paclitaxel versus placebo plus paclitaxel as first-line therapy for HER2-negative metastatic breast cancer (MERiDiAN): A double-blind placebo-controlled randomised phase III trial with prospective biomarker evaluation. Eur J Cancer 70: 146-155, 2017.

12 O'Connor JP, Jackson A, Parker GJ and Jayson GC: DCE-MRI biomarkers in the clinical evaluation of antiangiogenic and vascular disrupting agents. Br J Cancer 96: 189-195, 2007.

13 Preda A, Novikov V, Moglich M, Turetschek K, Shames DM, Brasch RC, Cavagna FM and Roberts TP: MRI monitoring of Avastin antiangiogenesis therapy using B22956/1, a new blood pool contrast agent, in an experimental model of human cancer. J Magn Reson Imaging 20: 865-873, 2004.

14 Padhani AR: Dynamic contrast-enhanced MRI in clinical oncology: Current status and future directions. J Magn Reson Imaging 16: 407-422, 2002.
15 Checkley D, Tessier JJ, Kendrew J, Waterton JC and Wedge SR: Use of dynamic contrast-enhanced MRI to evaluate acute treatment with ZD6474, a VEGF signalling inhibitor, in PC-3 prostate tumours. Br J Cancer 89: 1889-1895, 2003.

16 Hirashima Y, Yamada Y, Tateishi U, Kato K, Miyake M, Horita Y, Akiyoshi K, Takashima A, Okita N, Takahari D, Nakajima T, Hamaguchi T, Shimada Y and Shirao K: Pharmacokinetic parameters from 3-Tesla DCE-MRI as surrogate biomarkers of antitumor effects of bevacizumab plus FOLFIRI in colorectal cancer with liver metastasis. Int J Cancer 130: 2359-2365, 2012.

17 Miles DW, Diéras V, Cortés J, Duenne AA, Yi J and O'Shaughnessy J: First-line bevacizumab in combination with chemotherapy for HER2-negative metastatic breast cancer: pooled and subgroup analyses of data from 2447 patients. Ann Oncol 24: 2773-2380, 2013.

18 Miller KD, Chap LI, Holmes FA, Cobleigh MA, Marcom PK, Fehrenbacher L, Dickler M, Overmoyer BA, Reimann JD, Sing AP, Langmuir V and Rugo HS: Randomized phase III trial of capecitabine compared with bevacizumab plus capecitabine in patients with previously treated metastatic breast cancer. J Clin Oncol 23: 792-799, 2005.

19 Lambrechts D, Lenz HJ, de Haas S, Carmeliet P and Scherer SJ: Markers of response for the antiangiogenic agent bevacizumab. J Clin Oncol 31: 1219-1230, 2013.

20 Maru D, Venook AP and Ellis LM: Predictive biomarkers for bevacizumab: Are we there yet? Clin Cancer Res 19: 2824-2827, 2013.

21 Cheung YC, Chen SC, Su MY, See LC, Hsueh S, Chang HK, Lin YC and Tsai CS: Monitoring the size and response of locally advanced breast cancers to neoadjuvant chemotherapy (weekly paclitaxel and epirubicin) with serial enhanced MRI. Breast Cancer Res Treat 78: 51-58, 2003.

22 Li SP, Makris A, Beresford MJ, Taylor NJ, Ah-See MW, Stirling JJ, d'Arcy JA, Collins DJ, Kozarski R and Padhani AR: Use of dynamic contrast-enhanced MR imaging to predict survival in patients with primary breast cancer undergoing neoadjuvant chemotherapy. Radiology 260: 68-78, 2011.

Received June 27, 2018

Revised July 13, 2018

Accepted July 17, 2018 